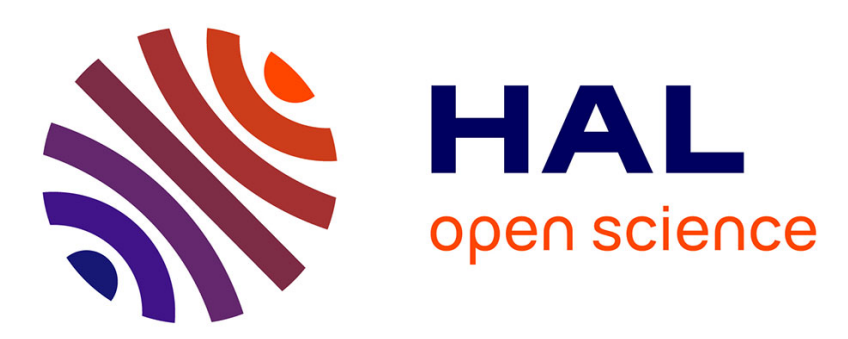

\title{
Les valeurs en éducation physique à l'école élémentaire et la morale sportive
}

Antoine Thépaut

\section{To cite this version:}

Antoine Thépaut. Les valeurs en éducation physique à l'école élémentaire et la morale sportive. Spirale - Revue de Recherches en Éducation, 1998, 22, pp.225 - 236. 10.3406/spira.1998.1585 . hal-03186970

\section{HAL Id: hal-03186970 \\ https://hal.univ-lille.fr/hal-03186970}

Submitted on 31 Mar 2021

HAL is a multi-disciplinary open access archive for the deposit and dissemination of scientific research documents, whether they are published or not. The documents may come from teaching and research institutions in France or abroad, or from public or private research centers.
L'archive ouverte pluridisciplinaire HAL, est destinée au dépôt et à la diffusion de documents scientifiques de niveau recherche, publiés ou non, émanant des établissements d'enseignement et de recherche français ou étrangers, des laboratoires publics ou privés. 


\section{Les valeurs en éducation physique à l'école élémentaire et la} morale sportive

Antoine Thépaut

\section{Citer ce document / Cite this document :}

Thépaut Antoine. Les valeurs en éducation physique à l'école élémentaire et la morale sportive. In: Spirale. Revue de recherches en éducation, $n^{\circ} 22,1998$. Les valeurs en éducation et en formation (2) pp. 225-236;

doi : https://doi.org/10.3406/spira.1998.1585

https://www.persee.fr/doc/spira_0994-3722_1998_num_22_1_1585

Fichier pdf généré le 31/03/2018 


\title{
Résumé
}

Peut-on former les élèves aux règles de la morale sportive dans le cadre des séances d'E. P. S à l'école élémentaire ? L'étude de la question fait apparaître que cette éducation est concomitante de l'acquisition des habiletés propres à chaque A. P. S. Nous avons cherché à relever dans le discours de quelques enseignants, quelles valeurs morales ils attribuent à cette discipline. L'étude d'une situation concrète permet de montrer comment l'enseignement d'une habileté en sport collectif peut être l'occasion d'une transmission de valeurs morales. Elle met en évidence qu'une perception moralisatrice du jeu peut aller à l'encontre de l'esprit même de ce jeu et amener l'enseignant à proposer des situations d'apprentissage inadaptées.

\begin{abstract}
Can one teach the rules of the moral standards of sport to children in the framewotk of P. E. classes in primary school ? As a result of studying this question it becomes evident that this education is concomitant with the acquisition of the skills peculiar to each sporting activity. We have sought to discern in the speech of a certain number of teachers moral values which they attribute to the sporting disciplines concerned. The studying of a real situation allows us to show how the teaching of a skill in a team sport can involve the transmission of moral values. This study highlights that a moral perception of a sport can be at odds wth the very spirit of the sport and lead the teacher to propose unsuitable teaching situations.
\end{abstract}




\section{LES VALEURS EN EDUCATION PHYSIQUE A L'ECOLE ELEMENTAIRE ET LA MORALE SPORTIVE}

Résumé : Peut-on former les élèves aux règles de la morale sportive dans le cadre des séances d'E.P.S à l'école élémentaire ? L'étude de la question fait apparaître que cette éducation est concomitante de l'acquisition des habiletés propres à chaque A.P.S. Nous avons cherché à relever dans le discours de quelques enseignants, quelles valeurs morales ils attribuent à cette discipline. L'étude d'une situation concrète permet de montrer comment l'enseignement d'une habileté en sport collectif peut être l'occasion d'une transmission de valeurs morales. Elle met en évidence qu'une perception moralisatrice du jeu peut aller à l'encontre de l'esprit même de ce jeu et amener l'enseignant à proposer des situations d'apprentissage inadaptées.

Mots-clés : Morale sportive - Éducation physique - École élémentaire.

\section{INTRODUCTION}

La pratique des activités physiques et sportives (A.P.S.), dans le cadre scolaire est traversée par la question des valeurs. Elle se situe à la croisée de deux institutions fondées sur la transmission de valeurs morales. L'institution scolaire qui, en se donnant pour mission d'éduquer les jeunes générations, se charge de transmettre les valeurs que la société souhaite voir intégrées par les enfants et les adolescents. L'institution sportive qui cherche à faire acquérir par ses pratiquants les valeurs auxquelles elle se réfère, c'està-dire, le plus souvent, celles de l'olympisme. Or, l'évolution actuelle du phénomène sportif interroge et plonge beaucoup d'éducateurs et d'enseignants dans le doute et la suspicion. La pratique sportive, souvent présentée comme une école de vie où s'apprennent le courage, la loyauté, l'amitié, le fair-play, ... se voit frappée d'opprobre. On découvre que des manifestations sportives sont truquées. La tricherie est parfois instituée en pratique organisée, la violence en fait systématique et banalisée pour s'adjuger la victoire à tout prix. Cette violence, véritable fléau des compétitions sportives, se manifeste tant à l'intérieur des stades, entre les joueurs eux-mêmes ou vis à vis de l'arbitre, qu'à l'extérieur, où certains spectateurs viennent au stade non 
pour soutenir leur équipe, encourager les sportifs, mais pour en découdre avec d'autres supporters, en colportant parfois des idéologies peu recommandables. On découvre également, ou on fait semblant de découvrir, que la pratique sportive est pilotée, structurée par l'argent qui, à lui seul attise toutes les convoitises et tous les vices. Bref, le sport, école de courage, d'amitié, de fraternité..., lieu de toutes les vertus, est aussi celui de la haine, de la tricherie, du nationalisme cocardier et de l'anti-intellectualisme. ${ }^{1}$ Lorsqu'un scandale éclate, le mouvement sportif, les médias, les hommes politiques s'en émeuvent et mettent en avance le mauvais exemple ainsi donné à la jeunesse. Le sport et la vie des sportifs a, dans l'idéologie commune, une valeur exemplaire pour la jeunesse. Toute entrave à cet exemple, tout écart de conduite est alors considéré comme un danger, un péril pour l'éducation vertueuse des jeunes générations.

S'il n'est pas question ici de faire l'apologie ou la condamnation du sport, ni une étude historique de l'évolution des valeurs et pratiques morales liées à l'activité physique, étude qui apparaitrait sans doute à bien des égards édifiante, on peut se demander, s'il est possible de former les jeunes aux valeurs de la morale sportive. Peut-on former aux règles de la morale sportive, dans le cadre des séances d'éducation physique et sportive (E.P.S.) à l'école élémentaire et plus particulièrement au Cycle 3 ? Peut-on, et à quelles conditions, faire de ces valeurs, un objet de savoir? Quelles valeurs et quelle morale peut-on transmettre par et à travers les A.P.S. ?

Les différents travaux, tant sur la question de la morale, qui ne peut pas être considérée comme un absolu, comme un système de règles intangibles s'imposant à tous les êtres humains, que ceux sur les questions d'éducation, ne permettent plus de penser la formation aux valeurs comme une intériorisation, inculcation des normes proposées par un adulte, un enseignant à des enfants, des élèves. On ne peut pas non plus considérer que cette éducation aux valeurs morales relève d'un processus de socialisation des jeunes entre eux, indépendamment de l'adulte, voire même à son insu, comme l'a soutenu Piaget (1932).

La situation scolaire, à l'école élémentaire, met en présence un maître et des élèves. Elle marque profondément l'expérience des enfants (Dubet et Martucelli 1996). A partir des travaux récents en sociologie, il est possible d'avancer que les conduites morales s'élaborent dans la relation des élèves avec le maître, par un travail d'ajustement permanent entre les attentes et conduites de l'adulte et celles des enfants. En E.P.S., cette formation ne peut donc s'envisager que dans l'interaction enseignant-élèves, à propos de l'en-

${ }^{1}$ Voir à ce sujet « Le sport c'est la guerre. » Le Monde diplomatique, Manière de voir $\mathrm{n}^{\circ} 30$, mai 1996.0 
seignement-apprentissage d'activités particulières que sont les activités physiques et sportives.

L'étude des travaux réalisés sur la morale mise en œuvre par les jeunes pratiquants dans les A.P.S., permet d'avancer que cette éducation morale passe par une formation aux A.P.S. Ainsi l'analyse des transgressions de la règle du jeu dans la pratique du handball et du football montre une différence importante entre la pratique des adultes et celle des enfants (Pfister 1987, Pfister, Roustan et Roustan 1991). Chez les adultes, les auteurs constatent une diminution importante des Interventions Adversives Non Conformes aux règlements (I.A.N.C.) de type non opératoire, c'est-à-dire non liées au déroulement même de la tâche motrice (altercation, dispute, insulte, bousculade...). Développer un niveau de jeu plus élaboré en football, handball, comme en tout jeu sportif collectif, passe par une diminution des I.A.N.C.. C'est mettre en œuvre des stratégies efficaces pour monter la balle dans l'espace de tir, ce qui nécessite de ne pas chercher à commettre volontairement une faute de contact sur un adversaire, mais plutôt à transmettre la balle à un partenaire, si le joueur est en difficulté face à un adversaire. Une diminution des I.A.N.C. passe en même temps et simultanément par une plus grande expérience de jeu, une plus grande habileté en jeu qui permet de mieux maitriser la charge émotionnelle de l'affrontement, charge qui est la source de ces I.A.N.C. chez les joueurs.

La formation aux valeurs de la morale sportive est selon nous indistincte de la formation à la pratique physique. Elle est concomitante de l'acquisition des habiletés propres à chaque A.P.S.. Elle passe par une confrontation aux problèmes réels que pose chaque A.P.S. Or, on peut penser que la logique scolaire, qui dans une logique d'apprentissage graduée, crée des problèmes formels, purement scolaires, produit en même temps une morale sportive purement formelle, une morale scolaire.

Analyser comment et quelles règles de la morale sportive s'enseignent et s'apprennent en E.P.S. nécessite d'étudier les interactions que se nouent entre le maître et les élèves à propos de l'enseignement, l'apprentissage des A.P.S.. C'est donc rechercher quelles conduites et comportements attendent les enseignants de leurs élèves d'une part, quelles conduites et comportements attendent les élèves de leur maître d'autre part.

A partir de l'étude des discours de quelques instituteurs et institutrices, on cherchera à identifier les valeurs qu'ils cherchent à transmettre, celles qu'ils attribuent à l'E.P.S.. Dans un second temps, on cherchera, par une étude de cas à soulever les problèmes que pose la référence aux valeurs morales dans l'apprentissage. Ceci permettra dans un troisième temps de soule- 
ver quelques hypothèses dans l'analyse de l'activité des élèves, et des rapports maîtres élèves à propos des valeurs morales en E.P.S.

\section{LES VALEURS VUES PAR LES ENSEIGNANTS}

Quelles valeurs accordent les instituteurs et institutrices à l'E.P.S à l'école élémentaire ? Quelles valeurs retiennent-ils ? Quels comportements attendent-ils des leurs élèves lorsqu'ils sont en éducation physique ? Ces valeurs ont été identifiées par l'analyse de contenu des discours tenus par des enseignants sur leur pratique d'enseignement de l'E.P.S. Il ne s'agit pas d'entretiens réalisés explicitement sur la question des valeurs en E.P.S, mais d'une analyse d'une enquête où les instituteurs étaient invités à décrire leur enseignement de cette discipline (Thépaut 1992).

Douze instituteurs et institutrices ont été interviewés. Ils ne constituent pas un échantillon statistique, mais ont été choisis selon leur profil. Les variables retenues pour le choix des enseignants étaient le sexe, (6 hommes, 6 femmes), l'implantation géographique et sociale des écoles, ( 6 enseignants situés dans des écoles des quartiers difficiles, 6 autres dans des écoles des quartiers bourgeois de la métropole lilloise), l'âge des enseignants (8 avaient entre 26 et 35 ans, 2 entre 36 et 45, 2 entre 46 et 55 ans.)

\section{Le rapport aux compétitions, aux rencontres U.S.E.P.}

Nous retenons ce thème en premier. La participation aux rencontres de l'Union Sportive de l'Enseignement Primaire (U.S.E.P.) apparaît en effet, comme l'élément le plus déterminant dans la conception, l'organisation et l'animation de l'E.P. ${ }^{2}$. Si d'autres A.P.S. sont proposées aux élèves par les enseignants, la participation aux rencontres U.S.E.P. détermine pour l'essentiel, de part son calendrier, la programmation des activités sur l'année. Elle délimite les objectifs de l'enseignement ; il s'agit d'être le plus rapidement possible, le plus performant dans l'activité considérée. Elle induit des normes - forme d'activités et règles du jeu — qui seront alors enseignées aux élèves dans le cadre des séance d'E.P.S. C'est enfin sur ce thème que les enseignants s'expriment spontanément le plus longuement, dans leur majorité, sur les valeurs de l'exercice physique.

\section{La convivialité}

Tous les enseignants s'accordent pour avancer que les rencontres de l'U.S.E.P. sont avant tout des moments conviviaux. Ils privilégient surtout l'aspect rencontre de leurs élèves avec les enfants des autres écoles de la cir-

\footnotetext{
${ }^{2}$ Tous les enseignants interrogés ne participent pas aux rencontres de l'U.S.E.P..
} 
conscription. Ils attendent de leurs élèves qu'ils soient heureux lors de ces rencontres, accueillants et ouverts sur les autres enfants.

\section{La compétition}

Les rencontres U.S.E.P. sont aussi des compétitions. Tous les enseignants également rejettent l'idée de compétition, qu'ils participent ou non à ces manifestations. Tous reconnaissent qu'elles ne doivent pas susciter, éveiller un esprit compétitif chez les élèves. L'idée de compétition contient la volonté de battre l'adversaire, de s'affirmer dominant. Elle comporte également les tricheries pour gagner à tout prix, la sélection des meilleurs élèves, les querelles de clocher d'école, la préparation à outrance et l'entraînement forcé pour amener les élèves de sa classe sur la plus haute marche du podium. Ce refus de la compétition semble autant marqué par le refus des attitudes répréhensibles qu'elle peut susciter chez les élèves que par la comparaison des résultats entre écoles sur un terrain déloyal puisqu'il ne semble pas y avoir au départ d'égalité des chances.

Deux attitudes distinctes apparaissent cependant dans les discours des enseignants : celle des instituteurs qui refusent de participer aux rencontres U.S.E.P. ou qui y participent mais en mettant en avance les effets pervers de la compétition., celles des instituteurs qui acceptent la compétition et lui reconnaissent une valeur formative.

\section{Le rapport à la règle}

Tous les enseignants interviewés attendent de leurs élèves qu'ils respectent les règles. L'E.P.S est présentée comme une discipline où l'on apprend à respecter la règle. Il apparaît alors deux types de règles. motrice.

Les règles $d u$ jeu, règles opératoires pour la réalisation de la tâche

La description des pratiques fait apparaître deux démarches. Les règles opératoires sont imposées par le maître. Dans un souci d'efficacité et pour aller plus vite dans la maîtrise de l'activité physique, il détermine seul les règles. Il attend que les élèves les appliquent à la lettre, qu'ils les appliquent et les connaissent rapidement pour pouvoir jouer. Les règles opératoires sont discutées, élaborées avec les élèves. Les enseignants attendent et souhaitent ici une participation des élèves, une collaboration de leur part. C'est ici une façon de procéder qui s'inscrit dans une démarche pédagogique d'élaboration des contenus et de construction des difficultés en fonction de l'activité des élèves. Les maîtres attendent également un meilleur respect des règles de la part des élèves dans la mesure où elles ont été négociées avec eux. 


\section{Les règles de fonctionnement de la classe en E.P.S. : le rapport à la discipline}

Tous les enseignants mentionnent et décrivent une discipline moins contraignante en E.P.S que dans la classe. Ils l'acceptent, en partie parce que « contraints et forcés » du fait même de la nature de l'activité où il est difficile d'interdire aux élèves de parler, de manifester leur joie, leur irritation d'être bousculé... et de la configuration spatiale (les enfants courent, se déplacent,..., comportements qui ne sont pas acceptés en classe.)

l'E.P.S est le lieu et le moment d'une relation différente avec l'enseignant. C'est un lieu, semble-t-il où les élèves se confient plus aisément. C'est aussi un moment où l'enseignant accepte davantage d'être contesté dans ses décisions et choix. Les élèves peuvent contester la réussite ou l'échec d'une action, les décisions prises sur le respect ou non des règles opératoires, surtout lorsque c'est l'enseignant qui arbitre.

\section{Le rapport à la mixité}

Les enseignants attendent ici de leurs élèves une bonne entente entre les filles et les garçons. L'E.P.S. provoque des situations inédites. En danse, par exemple, les garçons doivent être capables de donner la main à une fille. Il faut que les garçons acceptent de jouer avec les filles, mais surtout acceptent de les faire jouer : leur faire des passes, les laisser tirer au but et tenter de marquer. Enfin, certaines A.P.S. sont proposées pour permettre aux filles de se «sentir moins écrasées », d'être en position plus favorable par rapport aux garçons.

La mixité pose à l'école élémentaire surtout des problèmes de différence de niveau de jeu. Les maîtres attendent alors surtout que les garçons, plus avantagés par la nature même des activités et de leur finalité qui relèvent plus de la culture masculine, acceptent que les filles accèdent à leur monde, à leur univers, sans les ridiculiser dans leurs efforts méritoires.

\section{Autres valeurs}

D'autres valeurs attendues en E.P.S sont mentionnées par les instituteurs et institutrices, mais plus brièvement. Elles ne sont le fait que d'un ou deux enseignants pour chacune d'elle.

L'esprit d'équipe

La maîtrise de soi : Deux enseignants attendent de leurs élèves qu'ils maîtrisent leur violence et leurs comportements en E.P.S.

L'arbitrage : Deux enseignants indiquent confier l'arbitrage des rencontres de jeux collectifs aux élèves. Pour l'un, il s'agit d'apprendre aux élèves à arbitrer et à respecter l'arbitre. L'autre confie l'arbitrage aux élèves 
pour les jeux et sports collectifs dont il ne connait pas et ne maîtrise pas luimême les règles du jeu.

La responsabilité : Deux enseignants soulignent ici cette valeur. Le premier considère que les élèves doivent apprendre à se prendre en charge, participer à la mise en place du matériel et à l'arbitrage. L'E.P.S. a ici un rôle spécifique à jouer. L'autre, à l'inverse, refuse de déléguer ses responsabilités, considérant que les élèves de C.M. ne sont pas suffisamment autonomes pour cela.

\section{Interprétation}

Les enseignants disent spontanément peu de choses sur ce qu'ils attendent comme comportement moral de la part des élèves. Lorsqu'ils décrivent leur enseignement, ils s'attachent davantage à exposer les actions relatives aux activités physiques et en quoi ces actions déterminent le contenu et l'enchaînement des séances.

$\mathrm{Si}$ les A.P.S. sont communément présentées comme une éducation aux valeurs morales, notamment en référence aux valeurs de l'olympisme, la représentation qu'en ont les instituteurs et institutrices en est assez éloignée. La référence aux valeurs de l'olympisme n'apparaît pas comme une de leurs préoccupations.

L'éducation aux valeurs morales n'est pas une préoccupation essentielle. Elle ne détermine pas des formes d'enseignement spécifiques. Il apparaît que, si l'E.P.S participe à l'éducation morale des élèves, c'est davantage par la pratique elle-même, et non par des formes d'enseignement spécifiques. La pratique porterait en elle-même ses propres valeurs. Le simple fait de pratiquer vaut éducation.

La référence aux valeurs définies dans les Instructions Officielles est également peu présente dans le discours des enseignants. Seule la notion de responsabilité est citée. Elle est le fait de deux enseignants ; et en termes opposés. Pour l'un, l'E.P.S participe à la formation à la responsabilité. Pour l'autre, les élèves à cet âge ne sont pas suffisamment autonomes pour que l'on puisse leur confier des responsabilités.

L'élément qui apparaît les plus déterminant dans le discours des maîtres, est bien le rapport à la compétition. De ce point de vue, les enseignants s'accordent sur la valeur de la convivialité et rejettent dans leur majorité, les comportements et attitudes induits par l'esprit de compétition. En ce sens, ils sont plus proches d'une conception de l'activité physique développée par les filles et rejettent les conceptions développées par les garçons (Sabatier et Pfister 1995). Il y a là, pensons-nous, une source de malentendus entre les enseignants et les garçons. On peut penser que ces derniers sont en 
effet placés dans une position conflictuelle entre leur mode d'expression dans les activités physiques et les comportements qu'attendent d'eux les instituteurs.

Pour ce qui concerne le rapport à la règle, celui-ci doit, dans la représentation des maîtres, être unilatéral. Les élèves doivent respecter les règles du jeu parce qu'elle est la condition même d'un bon déroulement du jeu. Il y a là une conception adulte de la règle. Les règles doivent être respectées pour le « bien-être » de tous. Or, les travaux de Piaget (1932) et de Parlebas (1986) mettent en évidence, chez les enfants de cet âge, une période sensible et charnière dans l'accès à une morale autonome. Il y a là également une source de conflit permanent entre l'enseignant et les élèves, suscitant deux types de comportements chez ces derniers, entre la soumission aux demandes de l'adulte (morale hétéronome) et la recherche de discussion et de violation des règles. On peut ici, faire l'hypothèse que la distribution des élèves, entre ceux qui se soumettent et ceux qui contestent, ne se fait pas au hasard. Elle recoupe la distinction garçons/filles. Parmi les garçons, sans doute plus rebelles, plus compétiteurs, la distribution révèle, pensons-nous, le comportement scolaire de l'élève.

Enfin, les valeurs dépendent des orientations des enseignants. Le facteur le plus discriminant apparaît être ici, « la culture sportive de l'enseignant » (Thépaut 1992). Les variables sexe, âge ne distinguent pas les enseignants ; la composition sociale du public de l'école, à un degré moindre. Par contre, le rapport à la compétition sportive est d'autant plus rejeté que l'enseignant a une culture sportive faible ou à orientation ludique. Par contre, plus l'enseignant a une culture corporelle spécialisée, de type compétitif, et moins il se prononce contre la compétition.

En conclusion, les orientations prises par les instituteurs laissent entrevoir des sources de conflits, des zones d'incompréhension entre leur conception de l'activité physique et celle de certains élèves. Toutefois, il ne s'agit ici que d'orientations générales, exprimées à travers les discours que tiennent les enseignants sur leur pratique. L'observation directe peut permettre de repérer plus précisément ce qui s'enseigne.

\section{QUELLES PRATIQUES ? UNE ETUDE DE CAS}

L'analyse des discours que tiennent les enseignants sur leur pratique révèle ce qu'ils retiennent de réellement significatif. Elle permet de déceler leurs représentations qui orientent les pratiques d'enseignement. Toutefois, s'agissant des conduites morales, nous estimons que la formation aux règles de la morale sportive est concomitante de l'acquisition des habiletés de jeu. 
Or, l'essentiel des contenus d'enseignement est transmis au cours des séances (Marsenach 1991). On peut donc penser que c'est surtout dans le déroulement des séances que s'expriment les comportements autour desquels se construisent les conduites morales. Ici, seule l'observation détaillée permet de repérer ce qui est réellement enseigné. La description d'une étude de cas va permettre de dégager quelques hypothèses et de dessiner les lignes directrices de l'analyse.

L'observation de l'enseignement des jeux et sports collectifs à l'école élémentaire fait apparaître une centration importante sur l'échange de la balle entre deux partenaires : la passe. Aussi, lors des séances, les élèves sont fréquemment regroupés en deux équipes qui doivent s'affronter pour s'approprier la balle et aller tirer au but pour marquer (balle au capitaine, handball, basket-ball,...). L'observation courante amène alors à constater un regroupement des joueurs, partenaires et adversaires, autour du porteur de balle. Dans cette situation, les échanges sont ponctuels et bien souvent aléatoires. Ils sont plus le fait du joueur se débarrassant du ballon face à la pression rapprochée des adversaires comme des partenaires, qui tentent d'arracher le ballon des mains du porteur de balle. Les contacts sont nombreux, les situations conflictuelles fréquentes. Il est bien difficile de faire respecter les règles du non contact. Le ballon progresse difficilement vers la cible adverse. Les tentatives de tir sont souvent le fait d'un joueur seul, qui a réussi à se dégager de l'attroupement, par ses qualités physiques, la ruse ou son caractère. Ces joueurs, alors qualifiés de « joueurs individuels» ou «personnels », sont fréquemment rappelés à l'ordre par les maîtres, parce qu'ils ne jouent pas assez collectivement.

Pour remédier à cette forme précise du déroulement du jeu, les enseignants demandent aux élèves de se faire des passes. D'un problème de jeu, on en fait un problème de "morale sportive ». Les sports collectifs sont considérés avant tout comme des jeux d'équipe. Les enfants doivent apprendre à jouer collectivement, développer leur sens de la coopération. Ils doivent « se socialiser », entendu comme une bonne entente entre les joueurs et la capacité à se faire des passes. Or, le problème ici, est double.

La coopération, instituée en valeur, est assimilée à l'échange de la balle, alors que la « demande de passes » peut aller à l'encontre même de la logique de l'activité, logique qui vise à une progression rapide et efficace de la balle vers la cible adverse. Dans ce cadre, la passe qui n'est au départ qu'un moyen au service d'une fin, la progression de la balle vers la cible adverse, est instituée en finalité de l'enseignement des jeux et sports collectifs. Les aménagement de règles du jeu, de type «Faire une passe à l'ensemble des partenaires avant de tirer au but », avec l'objectif d'amener, d'obliger les 
joueurs à se faire des passes, peut provoquer des situations paradoxales où le joueur doit transmette la balle à un partenaire, sous peine de se voir sanctionné pour non respect de la règle, alors qu'il se trouve en position favorable de tir. Ces aménagements sont en ce sens inadaptés. Ils créent de « faux problèmes », qui font de l'E.P.S un enseignement artificiel, non fonctionnel et formaliste. La perception moralisatrice du jeu vient ici, à l'encontre même de l'esprit du jeu et de l'acquisition par les élèves de compétences fonctionnelles.

La question qui se pose alors, est de comment passer d'une morale de la coopération entendue comme la capacité/qualité à se faire des passes, à une coopération fonctionnelle entendue comme la participation de plusieurs joueurs à la réalisation d'un but commun, celui de faire progresser la balle vers la cible adverse pour tirer et marquer. C'est accepter pour chacun des joueurs de subordonner ses intérêts propres à la réalisation d'un projet collectif, dans le respect des règles du jeu, définissant les possibilités et contraintes de chaque joueur.

\section{LES VALEURS MISES EN GEUVRE PAR LES ENFANTS}

Quelques travaux ont été réalisés sur l'analyse des conduites morales mises en œuvre par les enfants dans la pratique des A.P.S. Les résultats de ces travaux sont à l'heure actuelle très contrastés. On peut relever une diminution des formules d'encouragement et de coopération chez les joueurs plus confirmés, en même temps qu'une augmentation des moqueries (Duret 1991). Plus l'enfant est jeune, plus le nombre de conduites agressives est élevé (Pfister, Roustan et Roustan 1991). Les garçons centrés sur une logique d'accomplissement et d'affirmation de soi, se manifestent par des conduites plus agressives. Les filles recherchent davantage le plaisir et de la relation (Sabatier et Pfister 1995).

Pour l'essentiel, la plupart de ces travaux concernent la pratique sportive hors de l'école. De plus, elles étudient essentiellement les relations des joueurs entre eux et non leurs interactions avec l'adulte, enseignant ou entraîneur. Une étude serait à conduire dans la situation scolaire, centrée sur les interactions entre le maître et les élèves afin de déterminer en quoi les conduites des uns conditionnent les conduites des autres, et vice versa. L'enjeu, ici, est de faire apparaître les logiques que mettent en œuvre les élèves dans leurs relations avec l'enseignant et avec les autres élèves afin de pouvoir identifier quelles conduites morales se constituent au cours des séances d'E.P.S. Elle nous renseignerait sur les conduites morales telles qu'elles se constituent au cours des séances. Cette discipline participe-t-elle à l'élabora- 
tion d'une morale sportive ? Elle nous renseignerait également sur le fonctionnement scolaire en mettant à jour ce qui se joue dans la relation maître élève à propos de l'enseignement, l'apprentissage des A.P.S.

\section{CONCLUSION}

Peut-on former aux valeurs de la morale ? Peut on former aux règles de la morale ? Peut-on, et à quelles conditions, faire des valeurs un objet de savoir? Les pratiques d'enseignement relevées ici, sont en relation avec les représentations qu'ont les instituteurs et institutrices au sujet de la pratique sportive et notamment la pratique compétitive. Elles sont en décalage avec la logique des sports collectifs. Il y a un refus de rechercher les déterminants de la réussite parce qu'ils renvoient aux déterminants de la victoire - le rapport au but et à la cible - qui provoquent des attitudes et des comportements de dépassement de l'autre, de domination, des comportements de type compétitifs. En ce sens, elles mettent à jour une des contradictions essentielles des A.P.S. de type compétitif dans leur rapport à la morale. Le joueur doit et ne peut donner le meilleur de lui-même qu'en cherchant à dominer, à s'imposer et battre son adversaire, ceci pour apprendre le sens de l'action collective, l'amitié... Apprendre à coopérer, à s'entendre dans et par la lutte, c'est là toute l'ambiguïté des jeux sportifs collectifs. C'est ce qui fonde, comme le rappellait $\mathrm{B}$. Jeu, l'importance de la fête, de la troisième mitemps. C'est la réconciliation après l'affrontement. C'est aussi l'échange des maillots qui fait que l'on ne sait plus qui est qui, qui est le vainqueur, qui est le perdant. "Les hiérarchies, et c'était pourtant la raison d'être de la compétition, sont brusquement supprimées »(Jeu 1977). Quelles formes de réconciliation peut-on concevoir à l'école élémentaire ?

\section{Antoine THÉPAUT Université Lille 2}

\footnotetext{
Abstract : Can one teach the rules of the moral standards of sport to children in the framewotk of P.E. classes in primary school ? As a result of studying this question it becomes evident that this education is concomitant with the acquisition of the skills peculiar to each sporting activity. We have sought to discern in the speech of a certain number of teachers moral values which they attribute to the sporting disciplines concerned. The studying of a real situation allows us to show how the teaching of a skill in a team sport can involve the transmission of moral values. This study highlights that a moral perception of a sport can be at odds wth the very spirit of the sport and lead the teacher to propose unsuitable teaching situations.
} 
Key-words : Moral standards of sport - Physical education - Primary school.

\section{Bibliographie}

Dubet F. \& Martuccelli D. (1996) A l'école. Sociologie de l'expérience scolaire. Paris, Seuil.

Jeu B. (1977) Le sport, l'émotion, l'espace. Paris, Vigot.

Marsenach J. et al. (1991), Éducation Physique et Sportive, quel enseignement ? Paris, I.N.R.P..

Parlebas P. (1986) Éléments de sociologie du sport. Paris, PUF.

Pfister R. (1987) «Étude des interactions agressives dans la pratique du football. Aspects temporels et topographiques. ». In Actes du colloque «Corps-Espace-Temps» (145-155). Paris, STAPS.

Pfister R., Roustan C \& Roustan R. (1991) « Les comportements d'agression en handball. ». In M. Laurent, J.F. Marini, R. Pfister et P. Therme (éds) Recherches en APS 3 (505-515). Paris, Actio UFR STAPS Marseille.

Piaget J. (1932) Le jugement moral chez l'enfant. Paris, PUF.

Sabatier C. \& Pfister R. (1995) «Transgression des règles par l'enfant dans la pratique du handball en milieu scolaire. » STAPS 36 (45-55).

Thépaut A. (1992) Culture corporelle et E.P.S. à l'école élémentaire : les paradoxes de l'initiation sportive. Mémoire de Maîtrise en Sciences de l'Éducation, Université Charles de Gaulle - Lille 3. 